\title{
Welchen Stellenwert hat die DVT in der HNO-Heilkunde?
}

D ie digitale Volumentomographie hält in den letzten Jahren zunehmend auch Einzug in die HNO-Heilkunde. Der Stellenwert im Vergleich zur Computertomographie wird nach wie vor kontrovers diskutiert.

An der Universitätsklinik für HNO, Kopf- und Halschirurgie in Marburg werden jährlich etwa 1.200 bis 1.400 Untersuchungen mit der digitalen Volumentomographie (DVT) durchgeführt, berichtete Dr. Christian Güldner. Damit hat die DVT einen wichtigen Stellenwert in der Routinediagnostik eingenommen. Das Konzept sei vielversprechend, „doch die Indikation muss stimmen“, betonte Güldner.

Mit der DVT (oder Cone Beam Computed Tomography, CBCT) werden hochaufgelöste Schnittbilder in allen Ebenen eines zylindrischen Volumens generiert. Knöcherne Strukturen können aufgrund des hohen Kontrastes zu Luft und Weichgewebe hervorragend dargestellt werden. Eine Differenzierung zwischen Weichgeweben ist jedoch nicht zuverlässig möglich. Pathologien der Gesichtsweichteile, endokranielle Komplikationen bei Traumata oder ein Verdacht auf Tumorerkrankungen können daher mittels DVT nicht abgeklärt werden.

In der Diagnostik der chronischen Rhinosinusitis tritt die DVT jedoch zunehmend in Konkurrenz zum Goldstandard Computertomographie (CT) und hat als gleichwertiges Untersuchungsverfahren Eingang in die aktuelle Leitlinie der Deutschen HNO-Gesellschaft

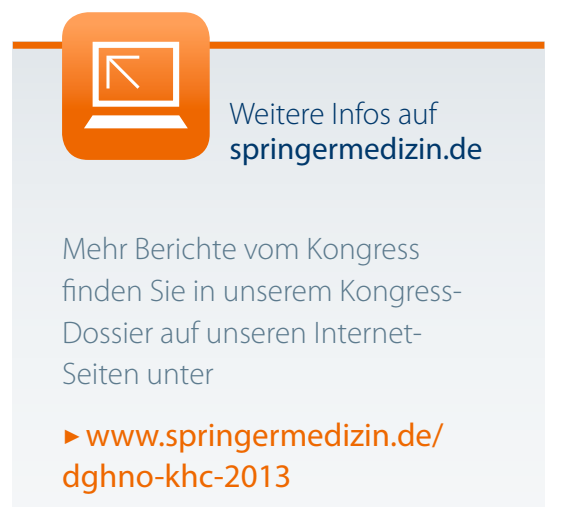

sowie die europäische Leitlinie gefunden. Sowohl Rhinobasis und Siebbein als auch die knöchernen Begrenzungen neurovaskulärer Strukturen können gut dargestellt werden. Ein weiterer Schwerpunkt der DVT sei die Lagekontrolle von Implantaten des Mittelund Innenohres sowie die Diagnostik der chronischen Otitis media, sagte Güldner.

Einer der Vorteile der DVT ist die Anwendung des Verfahrens im Rahmen der Teilgebietsradiologie durch den HNO-

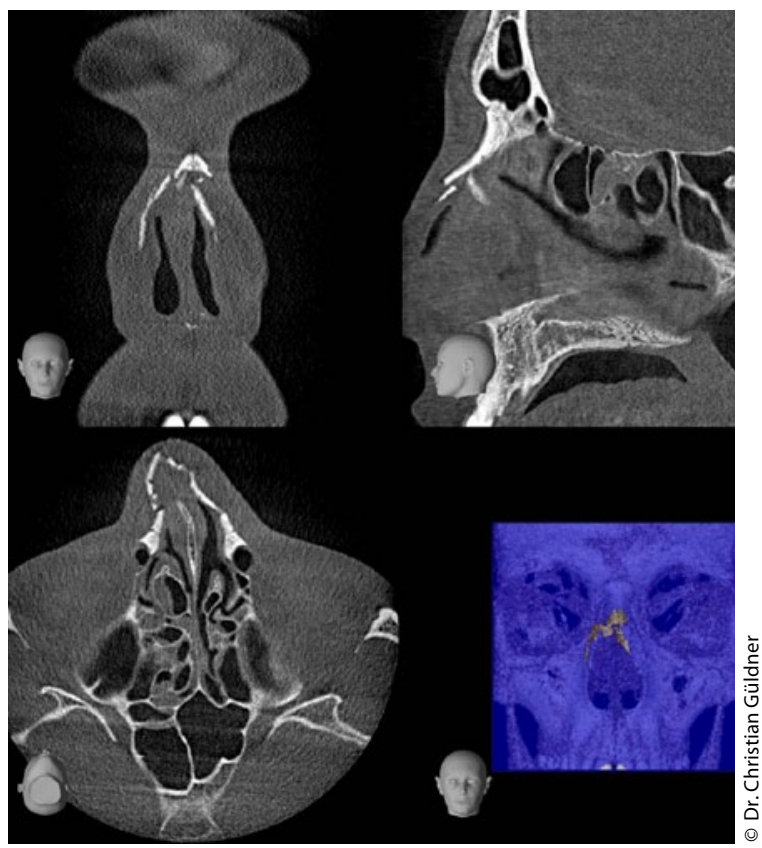

Bei einem klinischen Verdacht auf eine isolierte Nasenbeinfraktur konnte die DVT des Nasengerüstes die isolierte dislozierte Fraktur des Nasengerüstes bestätigen und somit die operative Therapie optimal vorbereiten. Arzt selbst. Dies ermögliche die optimale Verknüpfung von radiologischem und klinischem Befund sowie eine relevante Zeitersparnis für den Patienten, ergänzte Güldner.

In einer Fülle wissenschaftlicher Untersuchungen wird die geringere Strahlenbelastung durch die DVT gegenüber dem CT hervorgehoben. „Dabei werden jedoch nicht selten Äpfel mit Birnen verglichen“, kritisierte Prof. Dr. Michael Lell vom Radiologischen Institut des Universitätsklinikums Erlangen. Die mit DVT vs. CT untersuchten Indikationen oder der technische Entwicklungsstand der eingesetzten Geräte sei oft nicht vergleichbar. Während unterschiedliche CT-Geräte in der Mittelgesichtsdiagnostik gut vergleichbare Ergebnisse bei ähnlicher Dosis liefern, gibt es bei DVT-Geräten große Unterschiede in Dosis und Bildqualität, sagte Lell.

Durch geeignete Wahl der Aufnahmeparameter in der CT kann die Dosis deutlich, teilweise sogar unter das $\mathrm{Ni}$ veau der DVT-Standardprotokolle abgesenkt werden und es muss auf eine Beurteilung der Weichteilstrukturen nicht verzichtet werden. Von zentraler Bedeutung ist die klinische Fragestellung, an die die Untersuchung angepasst werden muss, betonte Lell. Bei

vielen Indikationen lassen sich mit Low-Dose-Protokollen diagnostisch ausreichende Ergebnisse erzielen.

Die kürzere Aufnahmezeit bei der CT gegenüber der DVT reduziert zudem die Wahrscheinlichkeit für Bewegungsunschärfen und damit das Risiko, dass eine Untersuchung wiederholt werden muss, was eine erneute Strahlenbelastung zur Folge hätte.

Bei der DVT wird derzeit ebenfalls überprüft, ob Dosierungen, die deutlich unter den herkömmlichen Standardprotokollen liegen, noch eine ausreichende Bildqualität gewährleisten.

Solange die Indikation richtig gestellt wird, sei die Strahlenexposition der modernen bildgebenden Verfahren schon heute wenig problematisch, betonte Lell. Die Belastung durch ein CT oder DVT der Nasennebenhöhlen entspricht etwa der von ein bis zwei Interkontinentalflügen.

Die Fortschritte in der Bildgebung lassen hoffen, dass sich die Strahlenbelastung für den Patienten bei hoher diagnostischer Sicherheit künftig weiter minimieren lässt. Angelika Bauer-Delto

Lell M, Güldner C: Streitgespräch: Stellenwert der Digitalen Volumentomographie in der HNO-Heilkunde. 